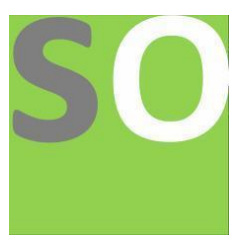

Article title: The Morbid Equation of Quantum Numbers

Authors: XD Dongfang[1]

Affiliations: Wutong Mountain National Forest Park[1]

Orcid ids: 0000-0002-3644-5170[1]

Contact e-mail: xddongfang@gmail.com

License information: This work has been published open access under Creative Commons Attribution License http://creativecommons.org/licenses/by/4.0/, which permits unrestricted use, distribution, and reproduction in any medium, provided the original work is properly cited. Conditions, terms of use and publishing policy can be found at https://www.scienceopen.com/.

Preprint statement: This article is a preprint and has not been peer-reviewed, under consideration and submitted to ScienceOpen Preprints for open peer review.

DOI: 10.14293/S2199-1006.1.SOR-.PP61CUI.v1

Preprint first posted online: 21 December 2020

Keywords: Coulomb field; equivalent field; Schrödinger equation; quantized energy; incompatible quantum states 


\title{
The Morbid Equation of Quantum Numbers
}

\author{
X. D. Dongfang \\ Wutong Mountain National Forest Park, Shenzhen, China
}

\begin{abstract}
The quantum model of valence electron generation orbital penetration of alkali metal elements with unique stable structure is investigated. The electric field outside the atomic kernel is usually expressed by the Coulomb field of the point charge mode, and the composite electric field in atomic kernel can be equivalent to the electric field inside the sphere with uniform charge distribution or other electric fields without divergence point. The exact solutions of two Schrödinger equations for the bound state of the Coulomb field outside the atom and the binding state of the equivalent field inside the atom determine two different quantization energy formulas respectively. Here we show that the atomic kernel surface is the only common zero potential surface that can be selected. When the orbital penetration occurs, the law of conservation of energy requires that the two energy level formulas must have corresponding quantum numbers to make them equal. As a result, there is no solution to the quantum number equation, indicating that the two quantum states of the valence electron are incompatible. This irreconcilable contradiction shows that the quantized energy of quantum mechanics cannot absolutely satisfy the law of conservation of energy.
\end{abstract}

\section{INTRODUCTION}

In a broad sense, the so-called quantization law is essentially the discrete law of the motion and change of matter. It is generally believed that the macro discrete law is different from the microscopic discrete law. So whether there is a common description method between them is an interesting and worthy of extensive and indepth study[1]. Quantum mechanics has achieved great success in describing the law of micro motion, and quantum theory has been developing continuously. However, the scope of quantum theory has not been clearly defined[2]. The essence of quantum mechanics has been perplexing us, which should be one of the reasons for the slow progress of quantum theory of macro interaction.

It may be the key to reveal the essence of quantum mechanics that some difficult problems of quantum theory are solved. For example, according to Bohr Sommerfeld quantization condition, the minimum nontrivial angular momentum of the hydrogen atom should be $\hbar[3]$. However, according to the square eigenvalue $L^{2}=l(l+1) \hbar$ of angular momentum in quantum mechanics, the minimum nontrivial angular momentum of the hydrogen atom is $\sqrt{2} \hbar[4]$. If the angular momentum can be measured experimentally, is the minimum nontrivial angular momentum of the hydrogen atom observed experimentally $\hbar$ or $\sqrt{2} \hbar$ ? This is the most intuitive difficult problem of quantum mechanics. However, the Bohr model of the old quantum theory and the Schrödinger equation of quantum mechanics respectively need these two contradictory angular momentum values to derive the same energy level formula of the hydrogen atom[5], which is consistent with the experimental observation. Perhaps abstract and vague knowledge such as the so-called semiclassical quantum theory can easily be chosen as the reason to avoid the above contradiction, but in fact, the minimum non-zero angular momentum of hydrogen atom is exactly $\hbar$. It is very difficult to solve the above difficult problem of minimum nontrivial angular momentum from the essence of quantization. The seemingly accurate and complete the- ory of quantum mechanics actually hides various more subversive logical inconsistent factors. Only by discovering and eliminating the logical contradictions hidden in scientific theories can we promote the correct development of theories. The unitary principle is a universal principle to test the logical self consistency of natural science theories, the content is as follows: different metrics can be used to describe natural laws, and there is a certain transformation relationship between them. The natural laws themselves do not change because of the $d$ ifferent metrics. The results of the transformation from different mathematical forms of natural laws expressed in different metrics into the same metric must be the same as the inherent form under this metric, $1=1$, which means the transformation is unitary. Bohr quantization angular momentum and quantum mechanics quantization angular momentum constitute two metric, but they do not meet the unitary principle.

Based on the unitary principle, we propose the morbid equation of quantum numbers derived from the quantum model of valence electron orbital penetration of alkali metal elements. When the valence electron moves outside the atom kernel, the effective charge[6] of the atomic kernel is equivalent to the point charge, and the interaction force between the valence electron and the atomic kernel is described by the Coulomb force. In the case of orbital penetration, if the valence electron moves in the atomic kernel, the effective charge of the atomic kernel should not be equivalent to the point charge, otherwise the singularity will cause infinite electric field force and violate the natural law. Because in theory, valence electrons can reach the center of the atomic kernel, just as there is a particle in the center of a star. Although the electric field in the atomic kernel is complex, the charge of the atomic kernel can be equivalent to the spherical charge with uniform distribution. The formula of interaction force between the valence electron and the atomic kernel is similar to Hooke's law. The quantum behavior of valence electrons outside and inside the atomic kernel is described by two Schrödinger equations[7], and two dif- 
ferent forms of quantized energy level formulas are given. Considering the conservation of energy, two kinds of correlated quantum states have at least one specific quantum number respectively, which makes the two kinds of quantized energy equal, resulting in the morbid equation of quantum numbers without any real number solution. Briefly speaking, when a particle moves between two different fields, two correlated quantum states constitute two metrics. However, the two quantized energy does not conform to the unitary principle, which means that the law of conservation of energy is broken.

\section{THE MORBID EQUATION OF QUANTUM NUMBERS}

If an isolated star body with uniform mass distribution has a straight hole through the center of the sphere, and a particle emits into the hole, the particle will move back and forth along the straight line where the hole is located. When a particle is outside the sphere body, the formula of the interaction force between the particle and the star is the universal gravitation between the particle and a mass point whose mass is equivalently concentrated at the center of the ball. It is the inverse square law. In the sphere, the interaction between the particle and the star is directly proportional to the distance from the particle to the center of the ball, similar to the spring force.

The Coulomb force is similar to gravitation, and it is also the inverse square law. Suppose that there is a charged sphere with uniformly distributed charges, and a particle with opposite charge moves through the sphere. The electric field force on the particle outside the sphere is the Coulomb force, and the electric field force in the ball is proportional to the distance from the particle to the center of the ball. Alkali metal element is a stable structure with an atomic kernel and an extra valence electron in its outer layer. The atomic kernel can be regarded as a sphere with uniform charge distribution, and the valence electron motion may occur orbital penetration. The ratio $\kappa$ between the radius of the atomic kernel and the minimum radius of the atom satisfies the inequality $0<\kappa \leq 1$. The quantum number describing the quantization energy of the valence electron in the electric field inside the atomic kernel is a non negative integer $l$, and the quantum number $n$ describing the quantization energy of the valence electron in the Coulomb field outside the atomic kernel is a positive integer. When the orbital penetration occurs, the energy is still conserved, and the two quantized energy formulas must be equal, which gives the algebraic equation

$$
\left(3-\frac{\kappa}{n^{2}}\right) \frac{\sqrt{\kappa}}{2 l+3}=1
$$

Where $0<\kappa \leq 1, n \geq 1, l \geq 0$.

However, no matter what the quantum numbers $n$ and $l$ take, equation (1) does not hold in the domain of definition. The equation (1) is transformed into a cubic equation about $\kappa$ expressed by the quantum numbers $n$ and $l$,

$$
(\sqrt{\kappa})^{3}-3 n^{2} \sqrt{\kappa}+(3+2 l) n^{2}=0
$$

Cubic equation (2) has a real root and two imaginary roots. According to Fontana-Cardano formula[8, 9], the real number root is

$$
\kappa=2 n^{2}+\frac{2^{\frac{2}{3}}+n^{4}\left[(3+2 l)^{2}-2 n^{2}+(3+2 l) \sqrt{(3+2 l)^{2}-4 n^{2}}\right]^{\frac{2}{3}}}{2^{\frac{1}{3}}\left[(3+2 l)^{2}-2 n^{2}+(3+2 l) \sqrt{(3+2 l)^{2}-4 n^{2}}\right]^{\frac{1}{3}}}
$$

The definition domain of the quadratic radical in the above formula is $(3+2 l)^{2} \geq 4 n^{2}$, that is, the two quantum numbers must satisfy the relation $n \leq l+3 / 2$. Because $\kappa$ is not only the increasing function of $n$, but also the increasing function of $l$. In order to find the minimum value of $\kappa$, when $n$ takes the minimum value, $l$ should also take the minimum value. Take the minimum quantum number $n=1$ and $l=0$ to get the minimum value of $\kappa$

$$
\kappa_{\min }=2+\left(\frac{7}{2}+\frac{3 \sqrt{5}}{2}\right)^{\frac{1}{3}}+\left(\frac{7}{2}-\frac{3 \sqrt{5}}{2}\right)^{\frac{1}{3}}
$$

The result is inconsistent with the domain $\kappa \in(0,1]$.

Equation (1) is called the morbid equation of quantum numbers. It means that the quantized energy of different bound states violates the law of conservation of energy when the same particle passes through different fields with boundaries. This contradiction is irreconcilable within the existing framework of quantum mechanics theory, and may need a new theory to solve it. The morbid equation of quantum numbers reveals that the great success of quantum mechanics has always implied sharp contradictions which must be corrected.

\section{DERIVATION OF MORBID EQUATION OF QUANTUM NUMBERS}

The effective charge number of alkali metal and other elements is represented by $Z^{*}$, and the elementary charge is represented by $e$. According to the description in the previous section, the electric field in the atomic kernel is equivalent to that of the positive charge $Z^{*} e$ uniformly distributed in the sphere with radius $\delta$. When the electron moves outside the atom kernel, the alkali metal atom forms the hydrogen like atom model. The radius $\delta$ of the atomic kernel does not exceed the Bohr radius $a_{0}=\varepsilon_{0} h^{2} / \pi m Z^{*} e^{2}$ of the hydrogen like atom. Where $\varepsilon_{0}$ is the dielectric constant, $h$ is the Planck constant, $\mathrm{mm}$ is the mass of the electron, $0<\kappa \leq 1$. The radius of the atom kernel is expressed as $\delta=\kappa a_{0}$, and its concrete form is

$$
\delta=\frac{\kappa \varepsilon_{0} h^{2}}{\pi m Z^{*} e^{2}}
$$


The effective charge density of the uniformly distributed effective charge of the equivalent sphere is $\rho=$ $Z^{*} e\left(4 \pi \delta^{3} / 3\right)^{-1}$, and the effective charge of the concentric sphere with $r \leq \delta$ is $q=\rho\left(4 \pi r^{3} / 3\right)=Z^{*} e r^{3} / \delta^{3}$. According to the Gauss theorem of electrostatic field, the electric field inside the atomic kernel of $r \leq \delta$ is $E_{1}=Z^{*} e r / 4 \pi \varepsilon_{0} \delta^{3}$, and that of the atom with $r \geq \delta$ is $E_{2}=Z^{*} e / 4 \pi \varepsilon_{0} r^{2}$. The electric field force on the electron is $F=e E$, and its direction points to the center of the sphere. Considering the penetration of valence electron orbit, let the common zero potential surface outside and inside the atomic kernel be a concentric sphere surface with $r=a_{c}$. according to the definition of potential energy $U=\int_{r}^{a_{c}} \mathbf{F} \cdot d \mathbf{r}$, the potential energies of the electron and the atomic kernel are calculated as follows

$$
\begin{aligned}
U_{1} & =\frac{Z^{*} e^{2} r^{2}}{8 \pi \varepsilon_{0} a_{c}^{3}}-\frac{Z^{*} e^{2}}{8 \pi \varepsilon_{0} a_{c}} \quad(r \leqslant \delta) \\
U_{2} & =\frac{Z^{*} e^{2}}{4 \pi \varepsilon_{0} a_{c}}-\frac{Z^{*} e^{2}}{4 \pi \varepsilon_{0} r} \quad(r \geqslant \delta)
\end{aligned}
$$

When the velocity of the valence electron arriving at the sphere surface of atomic kernel $(r=\delta)$ is $v$, it is obtained according to the energy conservation law of classical mechanics

$$
\frac{1}{2} m v^{2}+\frac{Z^{*} e^{2} \delta^{2}}{8 \pi \varepsilon_{0} a_{c}^{3}}-\frac{Z^{*} e^{2}}{8 \pi \varepsilon_{0} a_{c}}=\frac{1}{2} m v^{2}+\frac{Z^{*} e^{2}}{4 \pi \varepsilon_{0} a_{c}}-\frac{Z^{*} e^{2}}{4 \pi \varepsilon_{0} \delta}
$$

This formula is simplified to obtain $2 a_{c}^{3}-3 \delta a_{c}^{2}+\delta^{3}=0$, that is, $\left(a_{c}-\delta\right)^{2}\left(2 a_{c}+\delta\right)=0$, is obtained by simplification. This cubic equation has two equal positive roots $a_{c}=\delta$ and one negative root $a_{c}=-\delta / 2$. The solution satisfying the physical meaning $a_{c}>0$ is $a_{c}=\delta$. Therefore, the zero potential energy surface can only be selected on the equivalent sphere surface of the atomic kernel. Thus, the two potential energy expressions (6) are transformed into

$$
\begin{array}{ll}
U_{1}=\frac{Z^{*} e^{2} r^{2}}{8 \pi \varepsilon_{0} \delta^{3}}-\frac{Z^{*} e^{2}}{8 \pi \varepsilon_{0} \delta} & (r \leq \delta) \\
U_{2}=\frac{Z^{*} e^{2}}{4 \pi \varepsilon_{0} \delta}-\frac{Z^{*} e^{2}}{4 \pi \varepsilon_{0} r} \quad(r \geq \delta)
\end{array}
$$

The wave functions $\psi_{1}$ and $\psi_{2}$ are used to describe the quantum states of valence electrons in and out of the atomic kernel respectively. The Schrödinger wave equations for $\psi_{1}$ and $\psi_{2}$ are respectively as following

$$
\begin{aligned}
& \left(-\frac{\hbar^{2}}{2 m} \nabla^{2}+\frac{Z^{*} e^{2} r^{2}}{8 \pi \varepsilon_{0} \delta^{3}}-\frac{Z^{*} e^{2}}{8 \pi \varepsilon_{0} \delta}\right) \psi_{1}=E_{1} \psi_{1} \quad(r \leqslant \delta) \\
& \left(-\frac{\hbar^{2}}{2 m} \nabla^{2}+\frac{Z^{*} e^{2}}{4 \pi \varepsilon_{0} \delta}-\frac{Z^{*} e^{2}}{4 \pi \varepsilon_{0} r}\right) \psi_{2}=E_{2} \psi_{2} \quad(r \geqslant \delta)
\end{aligned}
$$

The first equation is the Schrödinger equation of threedimensional like harmonic oscillator, and the second equation is the Schrödinger equation of three-dimensional like hydrogen atoms. The natural boundary condition is $\psi(r \rightarrow \infty)=0, \quad|\psi|(0<r<\infty) \neq \infty$. The exact solutions of the two wave equations satisfying the natural boundary conditions determines that the corresponding energies in the equations are quantized, and the two energy eigenvalues[10-12] are respectively

$$
\begin{array}{ll}
E_{1}=\left(l+\frac{3}{2}\right) \frac{h}{2 \pi} \sqrt{\frac{Z^{*} e^{2}}{4 \pi \varepsilon_{0} \delta^{3} m}}-\frac{Z^{*} e^{2}}{8 \pi \varepsilon_{0} \delta} & (l=0,1,2, \cdots) \\
E_{2}=\frac{Z^{*} e^{2}}{4 \pi \varepsilon_{0} \delta}-\frac{m Z^{* 2} e^{4}}{8 n^{2} \varepsilon_{0}^{2} h^{2}} & (n=1,2,3, \cdots)
\end{array}
$$

These two quantized energy expressions of alkali metal elements are different from the common expressions in textbooks, and both have a constant term. This is because the classical law of conservation of energy determines that the common zero potential energy surface can only be selected on the surface of the atomic kernel when the orbital penetration occurs.

When the valence electrons of an atom in a certain energy state switch between the outer and inner atomic orbits, the quantized energy is bound to be conserved. The simplest model is that when the valence electron is on the surface of the atom, the two quantized energies must be the same, that is, $E_{1}=E_{2}$. According to formula (9), the equation is obtained

$$
\left(l+\frac{3}{2}\right) \frac{h}{2 \pi} \sqrt{\frac{Z^{*} e^{2}}{4 \pi \varepsilon_{0} \delta^{3} m}}=\frac{3 Z^{*} e^{2}}{8 \pi \varepsilon_{0} \delta}-\frac{\mu Z^{* 2} e^{4}}{8 n^{2} \varepsilon_{0}^{2} h^{2}}
$$

By substituting formula (5) into the above formula, we can get

$$
\frac{2 l+3}{\sqrt{\kappa^{3}}}=\frac{3}{\kappa}-\frac{1}{n^{2}}
$$

In other words, the morbid equation of quantum numbers (1) is obtained.

In mathematics form, the limit cases of $\kappa=1, l=0$ and $n \rightarrow \infty$ can make equation (1) hold, but the solution of this limit case does not conform to the physical meaning. The two forms of quantized energy are monotone increasing functions of $n$ and $l$, and they are conserved. The two forms of corresponding energy are equal. When $n \rightarrow \infty$, there must be $l \rightarrow \infty$. However, $n \rightarrow \infty$ is beyond the atomic range, and $l \rightarrow \infty$ cannot be explained. Moreover, only when $n \rightarrow \infty$ and $l=0$, there is a limit solution $\kappa=1$ in mathematical form. These are all contradictory. Therefore, the limit cases of $n \rightarrow \infty, l=0$ and $\kappa=1$ are not special solutions of equation (1) in accordance with physical meaning. This detail also indirectly reminds us that there may be some differences between physical mathematics and pure mathematics that are usually ignored. On the other hand, when $0<\kappa<1$, the left side of equation (1) is the fraction while the right side is natural number 1 , which is obviously contradictory. Therefore, equation (1) has no physical solution in the domain of definition. If there is a sphere with uniformly distributed charges, the quantum behavior of a particle with opposite charge passing through the uniformly charged sphere also has various quantum morbid equations. The quantum morbid equation shows that the 
Schrödinger equations of the different bound states of the microscopic particles in the sphere and outside the sphere are incompatible, which naturally includes the Dirac equation.

The law of conservation of quantized energy requires that there is a corresponding quantum number $l$ for any quantum number $n$, which makes equation (1) hold. In fact, there is no set of quantum numbers $n$ and $l$ that have physical significance to satisfy this equation. The morbid equation of quantum numbers in one-dimensional motion is different. In addition, considering that the values of the two wave functions on the real surface of atoms should be equal, the morbid equation of wave function$\mathrm{s}$ can be derived. If there is a sphere of uniformly distributed charges, the quantum behavior of a particle with opposite charge passing through the uniformly charged sphere also has various quantum morbid equations. The quantum morbid equation shows that the Schrödinger equations of the different bound states of the microscopic particles in the sphere and outside the sphere are incompatible, which naturally includes the Dirac equation[1315]. In fact, the Dirac equation of harmonic oscillator has been avoided. The mathematical derivation methods of all kinds of quantum morbid equations are the same. If the morbid equation of quantum numbers is solved, other problems will not exist. All in all, only the morbid equation of quantum numbers is introduced here.

\section{CONCLUSIONS AND COMMENTS}

The unitary principle is of great significance to test and establish scientific theory. A complete physical theory not only conforms to the unitary principle locally, but also conforms to the unitary principle in the whole domain. Using the unitary principle, we can find many important problems hide in modern physics theory. The difficulties of quantization angular momentum and the morbid e- quation of quantum numbers show that the conservation law of energy is destroyed because of quantized energy. These are actually computational problems rather than philosophical speculative problems. Quantum mechanics is a computational science, and its paradoxes should be expressed by mathematical equations. It is not helpful for the progress and development of physics theory to express the questions of quantum mechanics abstractly or to limit the philosophical speculation to weaken the logical difficulties of quantum mechanics. Physical logic difficulties inevitably contain new physics mysteries, which need to be discovered by scientific calculation. Quantum mechanics has puzzled some great physicists. Feynman famously declared "I think I can safely say that nobody understands quantum mechanics." [16-18] Einstein put forward the theory of light quantum, but evaluated quantum mechanics "God does not play dice with the universe." [19] Nevertheless, the success of quantum mechanics is undeniable. Almost all physics problems are interrelated. If one of the most basic physics problem$\mathrm{s}$ is solved, other physics problems can be solved well. Over the past 30 years, we have devoted ourselves to the discovery and solution of the difficult basic problems in theoretical physics. We have learned that the effective breakthrough of physical theory must come from the discovery and solution of the contradictions implied in the past theories that can not be reconciled logically. There must be systematic solutions to seemingly intractable physical problems. It seems that quantum mechanics is often misunderstood. Some reports on the progress of quantum theory are even based on metaphysical thinking and tend to develop towards metaphysics. The main reason is that the essence of quantum mechanics has not been known. It is one of the effective breakthroughs to reveal the essence of quantum mechanics and realize the unification of macroscopic and microscopic quantization theories by eliminating the logical difficulties of quantum mechanics such as the inconsistency of quantization angular momentum and the morbid equation of quantum numbers.
[1] Frauchiger, D. \& Renner, R. Quantum theory cannot consistently describe the use of itself. Nature Communications 9, 3711, doi:10.1038/s41467-018-05739-8 (2018).

[2] Lazarovici, D. \& Hubert, M. How Quantum Mechanics can consistently describe the use of itself. Scientific Reports 9, 470, doi:10.1038/s41598-018-37535-1 (2019).

[3] Schwinger, J. Magnetic charge and the charge quantization condition. Physical Review D Particles \& Fields 12 (1975).

[4] Landau, L. D. \& Lifshitz, E. M. ANGULAR MOMENTUM. Quantum Mechanics 8, 82-101 (1977).

[5] H nsch, T. W., Schawlow, A. L. \& Series, G. W. The spectrum of atomic hydrogen. Scientific American 240, 94-111 (1979).

[6] Slater, J. C. Atomic Shielding Constants. Physical Review 36, 57-64 (1930).
[7] Schrödinger, E. Quantization as an Eigenvalue Problem. Wave Mechanics 2, 151-167 (1968).

[8] Neumark, S. Solution of cubic and quartic equations. Solution of Cubic \& Quartic Equations, 30 (1962).

[9] Wang, Z.-X. \& Guo, D.-R. Introduction to special functions. Beijing: Science Press 354, 383 (1979).

[10] Messiah, A. \& Schiff, L. I. Quantum mechanics. Vol. 643 (McGraw-Hill College, 1968).

[11] Boiteux, M. The three-dimensional hydrogen atom as a restricted four-dimensional harmonic oscillator. Physica 65, 381-395 (1973).

[12] Al-shara, S., Mahasneh, A. \& Al-Qararah, A. Solution of Time-Independent Schrödinger Equation for a TwoDimensional Quantum Harmonic Oscillator Using He's Homotopy Perturbation Method. Journal of Mathematics Research 3, 3 (2011). 
[13] Dirac, P. A. M. The principles of quantum mechanics. (Oxford university press, 1981).

[14] Greiner, W. Relativistic quantum mechanics. Vol. 2 (Springer, 2000).

[15] Chen, R. Correction to Solution of Dirac Equation. arXiv preprint arXiv:0908.4320 (2009).
[16] Silverman, M. P. More than one mystery : explorations in quantum interference. (Springer-Verlag, 1995).

[17] Griffiths, D. J. Introduction to Quantum Mechanics. American Journal of Physics 319, 90-91 (2005).

[18] Aharonov, Y. \& Rohrlich, D. Quantum paradoxes: quantum theory for the perplexed. (John Wiley \& Sons, 2008).

[19] Schiemann, G. God does not play dice. Einstein's Still Topical Critique of Quantum Mechanics. (2005). 